\title{
Metabolic fingerprints of human primary endothelial and fibroblast cells
}

\author{
Polona Žigon ${ }^{1} \cdot$ Katjuša Mrak-Poljšak $^{1} \cdot$ Katja Lakota $^{1} \cdot$ Matic Terčelj $^{1} \cdot$ \\ Saša Čučnik ${ }^{1,2} \cdot$ Matija Tomsic $^{1} \cdot$ Snezna Sodin-Semrl $^{1,3}$
}

Received: 5 November 2015/Accepted: 18 March 2016/Published online: 7 April 2016

(C) Springer Science+Business Media New York 2016

\begin{abstract}
Introduction Human primary cells originating from different locations within the body could differ greatly in their metabolic phenotypes, influencing both how they act during physiological/pathological processes and how susceptible/resistant they are to a variety of disease risk factors. A novel way to monitor cellular metabolism is through cell energetics assays, so we explored this approach with human primary cell types, as models of sclerotic disorders. Objectives In order to better understand pathophysiological processes at the cellular level, our goals were to measure metabolic pathway activities of endothelial cells and fibroblasts, and determine their metabolic phenotype profiles.

Methods Biolog Phenotype MicroArray ${ }^{\mathrm{TM}}$ technology was used for the first time to characterize metabolic phenotypes of diverse primary cells. These colorimetric assays enable detection of utilization of 367 specific biochemical substrates by human endothelial cells from the coronary
\end{abstract}

Polona Žigon and Katjuša Mrak-Poljšak shared first authorship

Electronic supplementary material The online version of this article (doi:10.1007/s11306-016-1024-7) contains supplementary material, which is available to authorized users.

Polona Žigon

polona.zigon@guest.arnes.si

1 Department of Rheumatology, University Medical Centre Ljubljana, Vodnikova 62, 1000 Ljubljana, Slovenia

2 Faculty of Pharmacy, Chair of Clinical Biochemistry, University of Ljubljana, Ljubljana, Slovenia

3 Faculty of Mathematics, Natural Sciences and Information Technology, University of Primorska, Koper, Slovenia artery (HCAEC), umbilical vein (HUVEC) and normal, healthy lung fibroblasts (NHLF).

Results Adenosine, inosine, D-mannose and dextrin were strongly utilized by all three cell types, comparable to glucose. Substrates metabolized solely by HCAEC were mannan, pectin, gelatin and prevalently tricarballylic acid. HUVEC did not show any uniquely metabolized substrates whereas NHLF exhibited strong utilization of sugars and carboxylic acids along with amino acids and peptides.

Conclusion Taken together, we show for the first time that this simple energetics assay platform enables metabolic characterization of primary cells and that each of the three human cell types examined gives a unique and distinguishable profile.

Keywords Cellular metabolism $\cdot$ Phenomics $\cdot$ Phenotype MicroArrays · OmniLog · Human primary cells

\section{Introduction}

Primary cells cultured from ex vivo isolated tissues originating from different spatial sources of the same organism may show specific phenotypes, including distinct fingerprints of cellular metabolism (Pearson 2007). One way to monitor cellular metabolism is through multiplexed cell energetics assays. A new approach explored in this study was to use Biolog Phenotype MicroArray ${ }^{\mathrm{TM}}$ technology to characterize cells for metabolically and bioenergeticallylinked phenotypes (Bochner et al. 2011; Putluri et al. 2011). By simultaneously measuring utilization of hundreds of substrates, this assay system can reveal unique and insightful information on metabolic pathway activities and cellular responses to nutrients, hormones, cytokines, ions, and anti-cancer agents. 
Systemic sclerosis (SSc) or scleroderma is a chronic, multiorgan autoimmune disease complicated by early vasculopathy and heart involvement (Boueiz et al. 2010; Hettema et al. 2008; Meune et al. 2010; Ngian et al. 2011) as well as progressive fibrosis of the skin and internal organs, including lungs (Kuwana et al. 2004; Reiseter et al. 2015). Thus, endothelial cells and fibroblasts are among the earliest and most affected cell types in SSc. The endothelium is heterogeneous depending on its vascular bed and tissue/embryological origin. Different endothelial cell phenotypes exhibit differential responses to changes in their environment and signaling (Rosenberg and Aird 1999). Thus, human endothelial cells derived from the umbilical vein (HUVEC) have a specific and intrinsic expression pattern of inflammatory molecules as compared to human coronary artery endothelial cells (HCAEC). Cardiovascular cells that contribute directly to atherosclerosis and cardiac dysfunction are known to exhibit metabolic flexibility, characterized by the ability to switch from generating ATP primarily through oxidative phosphorylation to using glycolysis as the predominant energy source, as well as to shift from one fuel source to another (Vallerie and Bornfeldt 2015). This flexibility occurs in endothelial cells, myeloid cells, and cardiomyocytes during normal development and physiology, and is thought to have evolved to protect cells with heightened energy demand from the increased oxidative stress that can be a result of elevated rates of oxidative phosphorylation (Galvan-Pena and O'Neill 2014). The cells shunt glucose to oxidative side branches of glycolysis (Eelen et al. 2015), to provide energy more rapidly (Galvan-Pena and O'Neill 2014), or to use the most abundant fuel available (Kolwicz et al. 2013). Studies support the concept that metabolic flexibility confers the advantage of ensuring ATP supplies for continual cardiac contraction under a variety of physiological conditions (Goodwin and Taegtmeyer 2000; Kaijser and Berglund 1992; Schonekess 1997; Wentz et al. 2010).

Cardiac metabolism also undergoes a reprogramming in response to pathological hypertrophy, characterized by increased reliance on glucose metabolism and decreased fatty acid oxidation (Kolwicz et al. 2013). The question then arises whether metabolic flexibility and dysfunction in vascular and cardiac cells themselves could contribute to cardiovascular pathologies. Since patients with SSc have an elevated risk for vasculopathy, our aim was to determine what kind of metabolism HCAEC exhibit in comparison to a standardly used, however less appropriate, HUVEC model.

In the past, we have shown that HCAEC have a higher responsiveness and susceptibility to cytokines, and can as such, represent an excellent gene and protein expression model for evaluating the effects of vascular stress (Lakota et al. 2013, 2007, 2009). Therefore, it is of interest whether
HCAEC have a different metabolic phenotype at baseline levels than HUVEC.

SSc is also a complex chronic connective tissue disease characterized by progressive fibrosis (Laar and Varga 2015) which can lead to irreversible damage of external and internal organs, such as the skin and lungs, respectively. The major cells implicated in fibrosis are fibroblasts, with their main physiological function in wound healing and tissue remodeling. The fibrotic component is dominant in SSc, as compared to other autoimmune diseases and determines its prognosis and therapeutic refractoriness. Studies in cultured SSc skin fibroblasts have facilitated the identification of potential pathways involved in their profibrotic phenotype. Profibrotic fibroblasts characterized by abnormal growth and extracellular matrix synthesis may differentiate or expand from normal resident fibroblasts and multiple factors, including signaling pathways appear to be involved in the development and/or persistence of the SSc fibroblast phenotype (Usategui et al. 2011). We postulate that fibroblasts, when activated/stimulated, under stress microenvironment conditions, could function much like cancer cells, with unbridled proliferation, due to a redirection of their metabolism by the reverse Warburg effect (Pavlides et al. 2010). In cancer, fibroblasts/stromal cells can convert to myofibroblasts, which (using glycolysis) can produce large amounts of lactate and pyruvate, thus feeding the faster growing cancer cells (Pavlides et al. 2009). It is interesting that in SSc, where fibroblasts are also highly activated and responsible for abnormal extracellular matrix accumulation (Usategui et al. 2011), inhibition of autophagy and aerobic glycolysis was suggested as a primary strategy to reverse fibrosis (Castello-Cros et al. 2011). Thus, studying metabolic utilization of substrates is relevant in disease development, as well as for providing innovative strategies to combat disease activity.

No data to our knowledge is currently available on the metabolic activity of human primary cell types using Biolog Phenotype MicroArray ${ }^{\mathrm{TM}}$ technology, and specifically fibroblast or endothelial cells utilizing multiple substrates. So, we aimed to measure metabolic activity of primary human endothelial and fibroblast cells on multiple carbon and energy sources, as well as determine their specific metabolic fingerprints using the Biolog Phenotype MicroArray $^{\mathrm{TM}}$ and OmniLog assay system.

\section{Materials and methods}

\subsection{Cell culture}

HCAEC, HUVEC and NHLF (normal human lung fibroblasts) are all primary cell types generated ex vivo from isolated tissue (Lonza, Inc. Basel, Switzerland). Cell 
characteristics as reported by the manufacturer are summarized in Supplementary Table 1. The cells were plated onto six well plates or $75 \mathrm{~cm}^{2}$ flasks (TPP, Trasadigen, Switzerland) at $37{ }^{\circ} \mathrm{C}$ in a humidified atmosphere with $5 \%$ $\mathrm{CO}_{2}$. HCAEC were grown in EGM-2 $\mathrm{M}$ medium containing $5 \%$ fetal bovine serum (FBS); for HUVEC we used EBM-2 medium containing $2 \%$ fetal bovine serum (both from Lonza Inc., Basel, Switzerland). NHLF were cultivated in FGM-2 medium containing $2 \%$ FBS. All media components were from Lonza Inc. (Basel, Switzerland). Prior to the experiments, cells were incubated in serum-free medium for $30 \mathrm{~min}$.

\subsection{Phenotype MicroArray and OmniLog optimization experiments}

HCAEC and NHLF specifically, were used for optimization experiments. Optimal cell medium, reagent dye and seeding cell densities were tested. Two different cell media were also tested, specifically IF-M1 (with amino acids) and IF-M2 (without amino acids) with two different Redox Dye Mixes MA and MB using initially three different seeding densities. All Phenotype MicroArray assay components were from Biolog Inc. (Hayward, CA USA).

Specifically, cells were seeded into 96 well plates (TPP, Trasadigen, Switzerland) at seeding densities of 20.000, $10.000,5.000,2.500,1.250$ and 625 cells/well in a compete medium MC-0 (50 $\mu \mathrm{L} /$ well). The MC-0 medium was prepared using either IF-M1 or IF-M2 medium with added Pen/Strep, L-glutamine $(0.3 \mathrm{mM})$ and dialyzed FBS (at final concentration $5 \%$ ) (all from Lonza Inc., Basel, Switzerland). Cells were then incubated for $1 \mathrm{~h}$ at $37{ }^{\circ} \mathrm{C}$ under $5 \% \mathrm{CO}_{2}$, before adding Biolog Redox Dye Mix MA $(10 \mu \mathrm{L})$ or Biolog Redox Dye Mix MB $(10 \mu \mathrm{L})$ and measuring tetrazolium reduction, resulting in formation of a purple colour.

\subsection{Tetrazolium colorimetric measurements}

All cell types (HCAEC, HUVEC and NHLF) for experiments on Biolog Phenotype MicroArray plates PM-M1 to PM-M4 (Biolog Inc., Hayward, CA, USA) were used between passages 4 and 5 . A total of 367 different carbon and energy substrates were tested, as shown in Table 1. Cells were initially grown in $75 \mathrm{~cm}^{2}$ culture flasks to confluency, before they were resuspended at a density of 200.000 cells/ml in Biolog IF-M1 medium with Pen/Strep, L-glutamine and dialyzed-FBS. Cells were then washed $2 \times$ with PBS and detached with trypsin at $37{ }^{\circ} \mathrm{C}$ for $2 \mathrm{~min}$. Trypsinization neutralizing solution was added and cells were centrifuged at $350 \times g$ for $5 \mathrm{~min}$ at room temperature. MC-0 assay medium was added and cell count determined on Countess cells counter (Thermo Fisher Scientific, USA).
Additional MC-0 was added to achieve a cell density of 10.000 cells $/ 50 \mu \mathrm{L}$, which was seeded into the plates and incubated at $37{ }^{\circ} \mathrm{C}$ under $5 \% \mathrm{CO}_{2}$ for $18 \mathrm{~h}$. Following the incubation, $10 \mu \mathrm{L}$ Redox Dye Mix MB/well was added, the plate covered with sealing tape to prevent $\mathrm{CO}_{2}$ loss and incubated at $37{ }^{\circ} \mathrm{C}$ in the OmniLog (Biolog Inc., Hayward, CA, USA) for $24 \mathrm{~h}$ to kinetically measure tetrazolium reduction. OmniLog measures the intensity of purple colour formation using a CCD camera to record digital images every $15 \mathrm{~min}$. All experiments on PM-M1 to PM-M4 plates were done in triplicate (48 plates total).

\subsection{Statistical analysis}

The Omnilog PMM Data Analyses Software was used to analyze metabolic phenotypes. The data were adjusted by subtracting average values of three negative control wells from all other samples at the end time point. Metabolic responses were normalized due to the differences in doubling times of the different cell types. Omnilog data exportable parameters were exported to Microsoft Excel and Bar graphs were made. Venn diagrams and digital images were processed using CorelDraw.

\section{Results and discussion}

\subsection{Primary human cell types and growth conditions}

In order to investigate cell morphology and growth of human primary endothelial (HCAEC, HUVEC), as well as fibroblast (NHLF) cells, inverted microscopy was used. Cells appeared phenotypically healthy, with endothelial cells exhibiting "cobblestone" morphology, while fibroblasts appeared typically elongated (Fig. 1). For measurement of the cellular metabolism Biolog Phenotype MicroArray was used. The kinetics of purple formazan accumulation was measured with OmniLog PMM System, operating as an incubator/reader that holds up to 50 microplates, and reads/quantifies colour density in each well, every 15 min over the user-specified time period. Prior to all measurements of cell metabolic activity, it is useful to first optimize which of the two dye mixes will work better for the cell type under study and how many cells will be needed per well to produce a given amount of reduced formazan product within an appropriate time frame. Dye preference and optimal number of cells for determining growth in complete medium has previously been determined for 15 different human, mouse and rat cell lines (http://biolog. com/pdf/pmmlit/00P\%20133rC\%20Redox\%20Dye\%20Mix\% 20Brochure\%20JUL07.pdf), however no data is currently available on optimal conditions for primary human endothelial and fibroblast cells. We therefore performed experiments which 


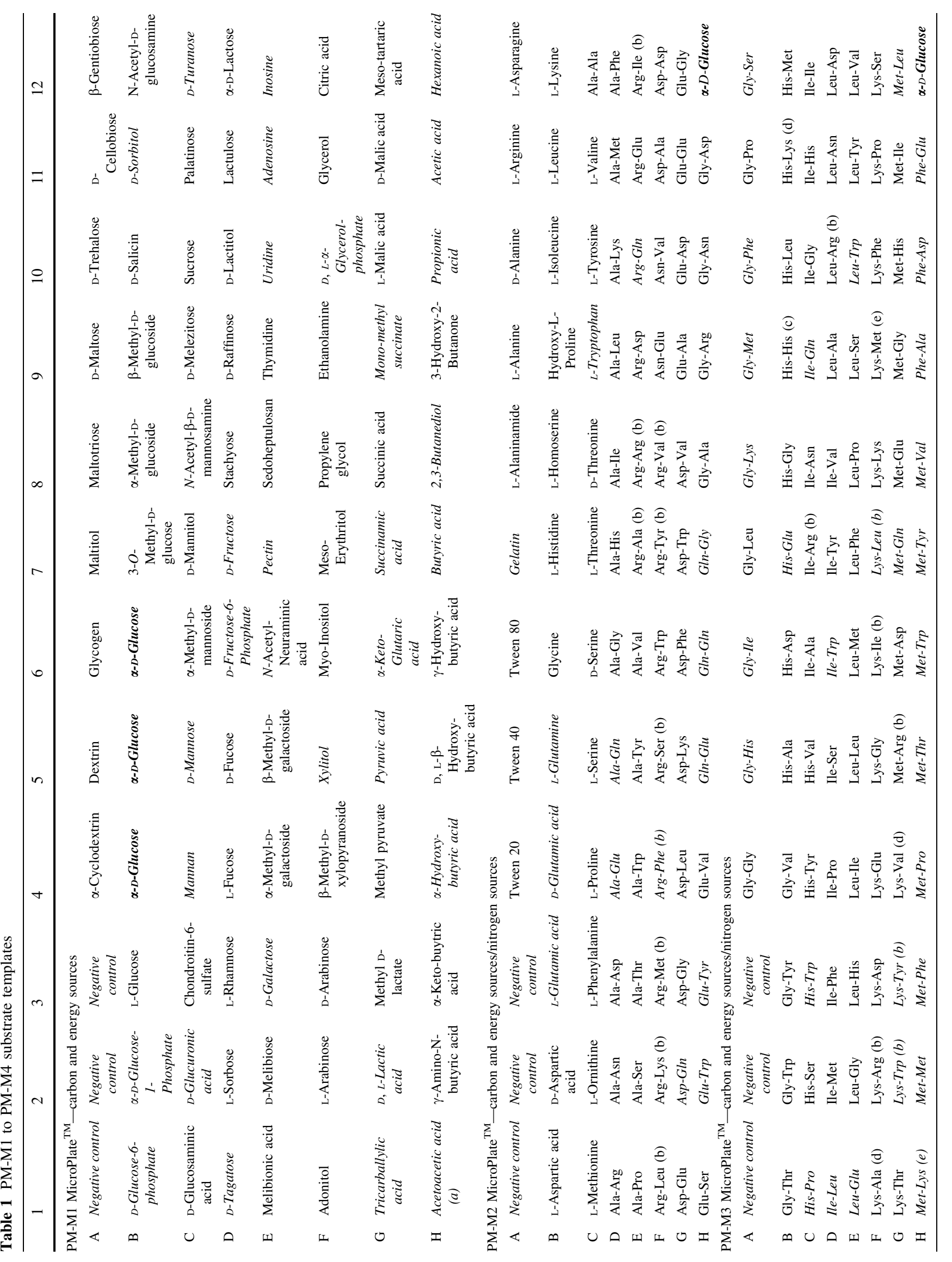




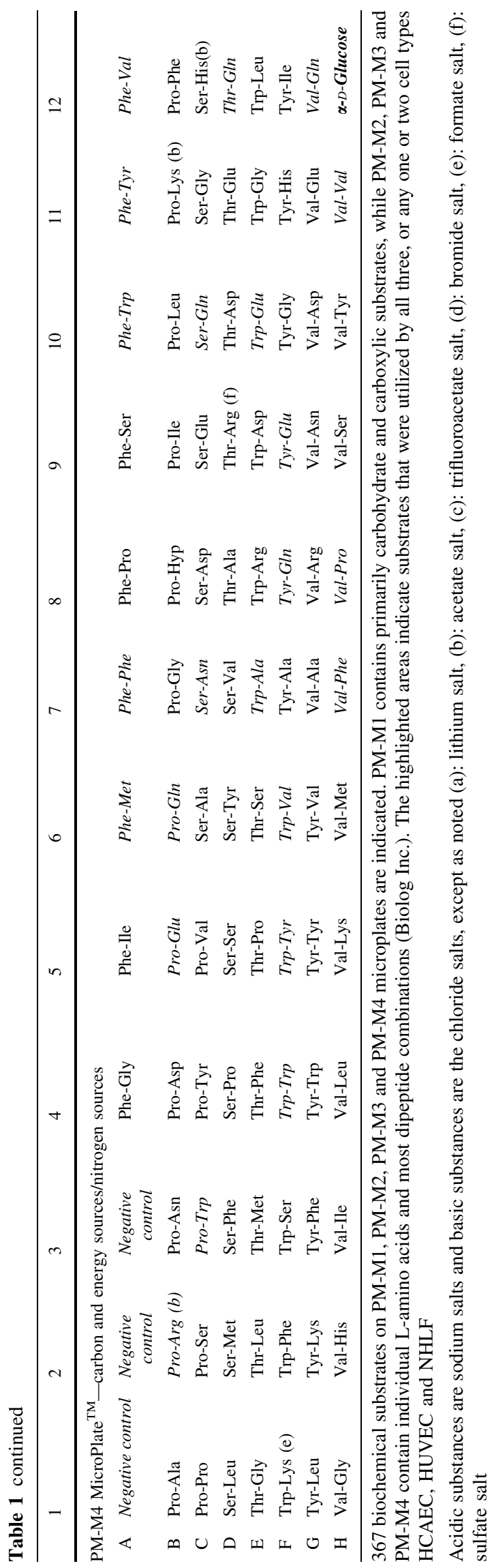

determined the specific media (M1 or M2), cell densities and Biolog redox dye mixes (MA or MB) to be used (Fig. 2). No reaction was detected using Biolog Redox Dye Mix MA. M1 medium enabled slightly stronger reactions compared to M2 medium. The cell density of 10.000 cells/well was the first dilution to give maximal readings while 5.000 cells/well showed considerably decreased responses. The optimal conditions for growth of HCAEC and NHLF were therefore set on 10.000 cells/well in Biolog IF-M1 medium with $1 \times$ Pen/Strep, $0.3 \mathrm{mM}$ L-glutamine and $5 \%$ dialysed FBS with Biolog Redox Dye Mix MB (10 $\mu \mathrm{l} / \mathrm{well})$ (Fig. 2). These conditions were used in all subsequent experiments determining cell growth on different substrates.

\subsection{Utilization of substrates on PM-M1, PM-M2, PM-M3 and PM-M4 microplates}

The extent to which human primary cell types from various tissues use different carbon substrates for energy has, to our knowledge, not been systematically investigated. It is known that, in addition to glucose, animal cells can metabolize and grow on other substrates (Bochner et al. 2011). In 1976, a survey of nutrient metabolism (Burns et al. 1976) examined 93 carbohydrates of which (a) 15 supported mammalian cell proliferation and (b) 42 were toxic or growth inhibitory and concluded that carbohydrate preferences of cells can be utilized to biochemically distinguish between different mammalian cell lines. In the following years, culture media were developed using glucose, pyruvate, and glutamine as energy sources, which were shown to support growth of most cell types and the interest to investigate the diversity of possible nutrients metabolized by different cell types waned. Recently, there is renewed interest to understand how the metabolism of different cell types could contribute to pathological pathways and help develop new approaches in nutritional therapy to support and improve treatment of a wide range of systemic disorders.

Using the optimized assay conditions we measured metabolic utilization of different substrates by HCAEC, HUVEC and NHLF in four microplates PM-M1 to PMM4 containing 367 substrate. Representative plates out of three independent experiments are shown (Fig. 3). Glucose is widely accepted as the primary nutrient for the maintenance and promotion of cell function and all cell types tested produced a strong response in wells containing glucose (Fig. 3, black boxes). Background, the average absorbance measured in negative controls, was subtracted from the data used for further analysis (Fig. 3, blue boxes). Exclusive substrates are indicated in HCAEC (Fig. 3, green boxes) and NHLF (yellow boxes), while HUVEC showed no exclusively utilized substrates. 


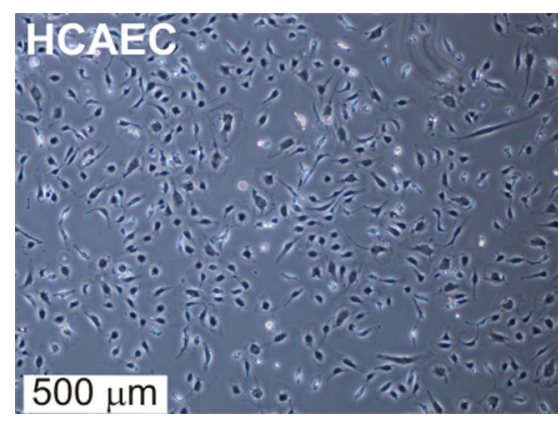

Fig. 1 Different phenotypes of human primary endothelial and fibroblast cells. Micrographs show normal, healthy endothelial cells isolated from coronary arteries, umbilical vein and fibroblasts from
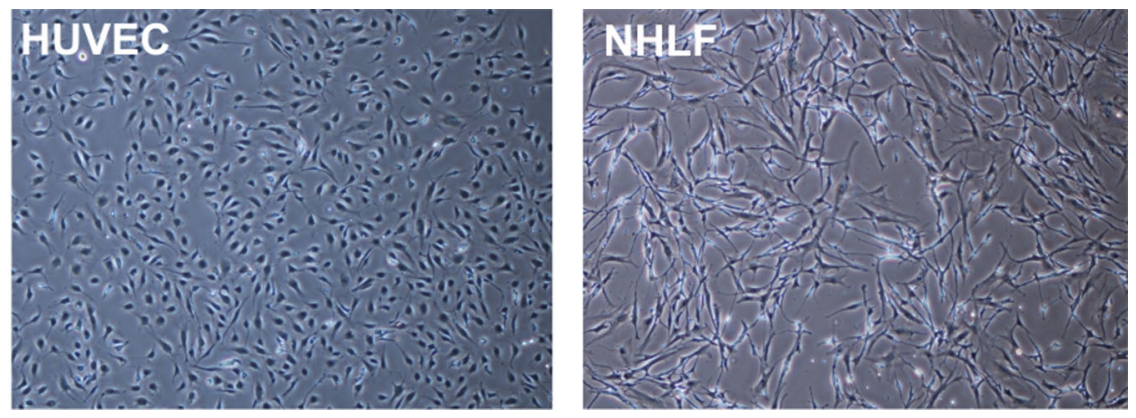

the lungs. HCAEC human coronary artery endothelial cells, HUVEC human umbilical vein endothelial cells and $N H L F$ normal human lung fibroblasts
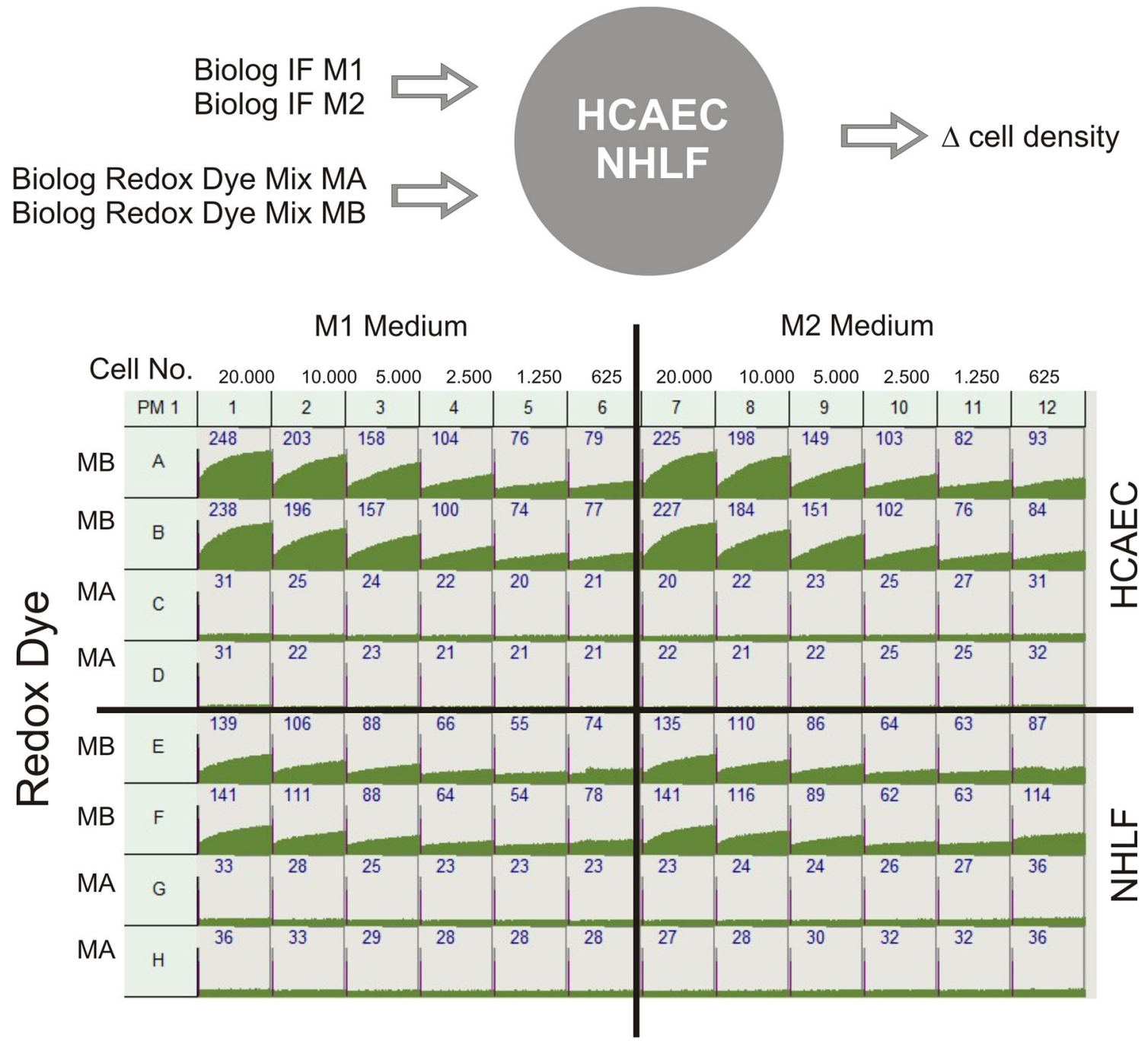

Fig. 2 Optimization of Biolog media, redox dye mixes and cell density, for $H C A E C$ human coronary artery endothelial cells and $N H L F$ normal human lung fibroblasts. The OmniLog reading results, as area under curve, of the separate wells, are presented in green 


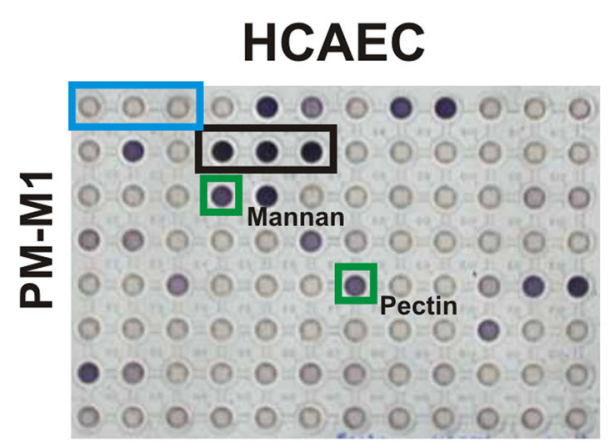

HUVEC
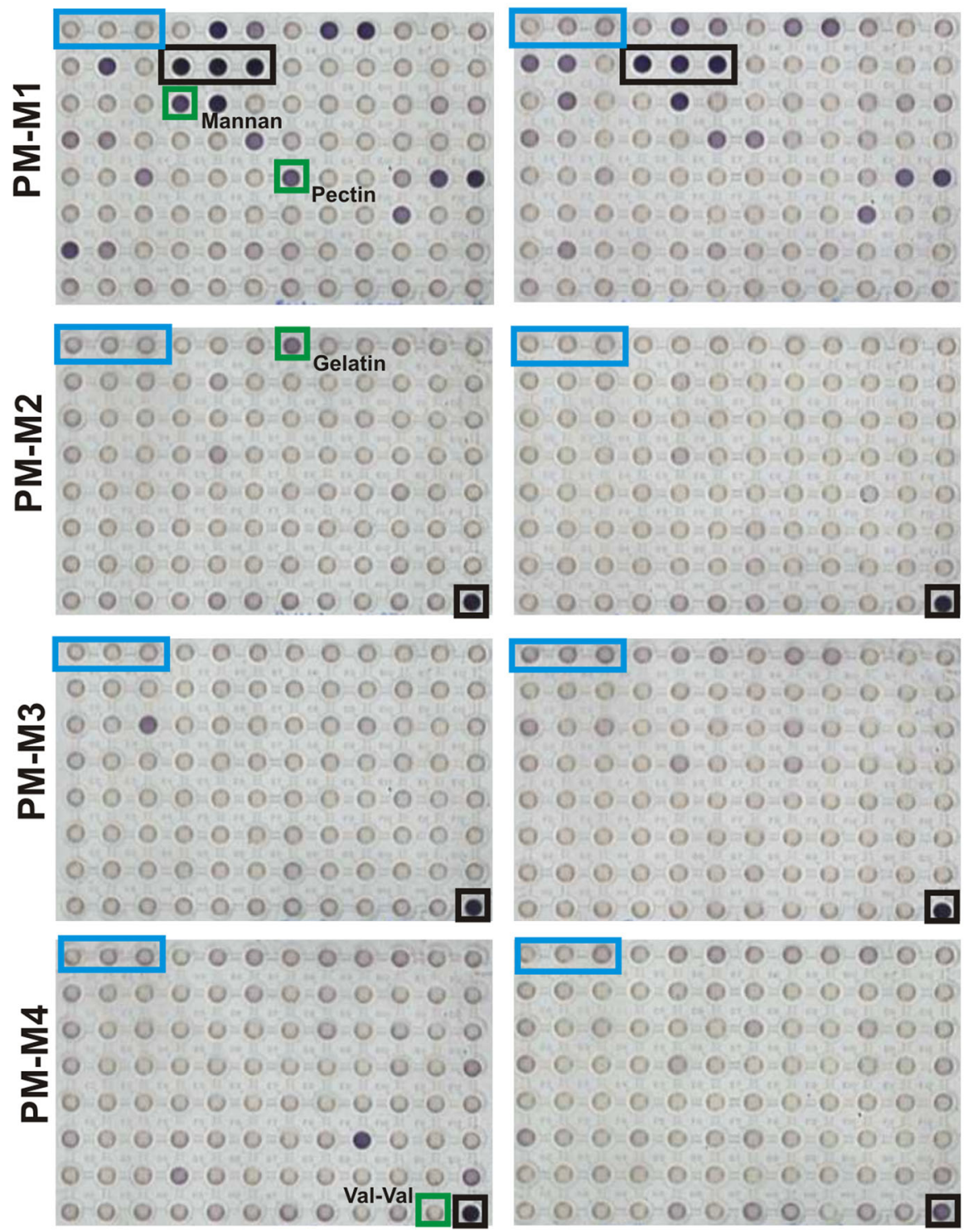

Fig. 3 HCAEC, HUVEC and NHLF assayed in PM-M1 to PM-M4. The columns show the endpoint of a $24 \mathrm{~h}$ incubation of a representative replicate of three independent experiments performed. Positive wells with glucose utilization are indicated with black boxes, while negative, background wells are shown with blue boxes. Exclusive

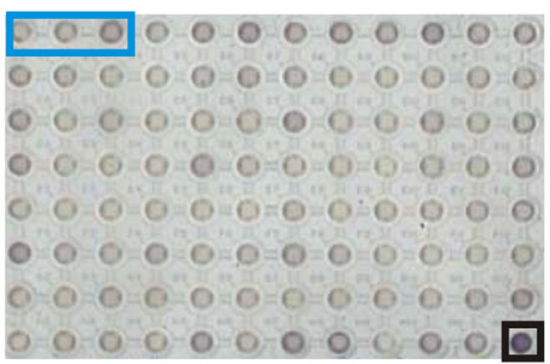

NHLF
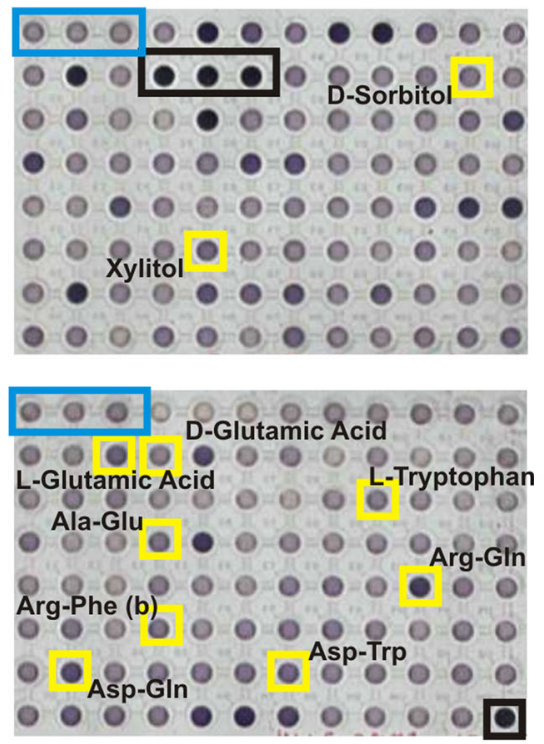

results obtained for HCAEC are shown in green boxes and for NHLF in yellow boxes. HCAEC human coronary artery endothelial cells, $H U V E C$ human umbilical vein endothelial cells and NHLF normal human lung fibroblasts

\subsection{Data analysis of substrate utilization}

All three cell types highly utilized adenosine, inosine, Dmannose and dextrin as substrates (to tetrazolium reduction levels above $100 \mathrm{mOD}$ ), comparable to glucose. Using plates PM-M1 through PM-M4, patterns of substrate utilization were observed for HCAEC, HUVEC and NHLF, as indicated in green, red and yellow bars, respectively (Fig. 4). HCAEC (green bars), prevalently utilized substrates on PM-M1 and to a lesser extent on PM-M3, with

only a few substrates utilized on PM-M2 and PM-M4. NHLF, on the other hand, showed high and exclusive utilization of certain glutamine-associated peptides on PMM2, PM-M3 and PM-M4, not observed in either HCAEC or HUVEC (Figs. 3, 4).

HCAEC were able to use a larger number of substrate nutrients, with higher tetrazolium reduction, as measured in mOD for energy production than HUVEC (such as dextrin, glycogen, maltotriose, D-maltose, $\alpha$-D-glucose-6-phosphate, mannan, D-mannose, D-turanose, D-fructose-6- 

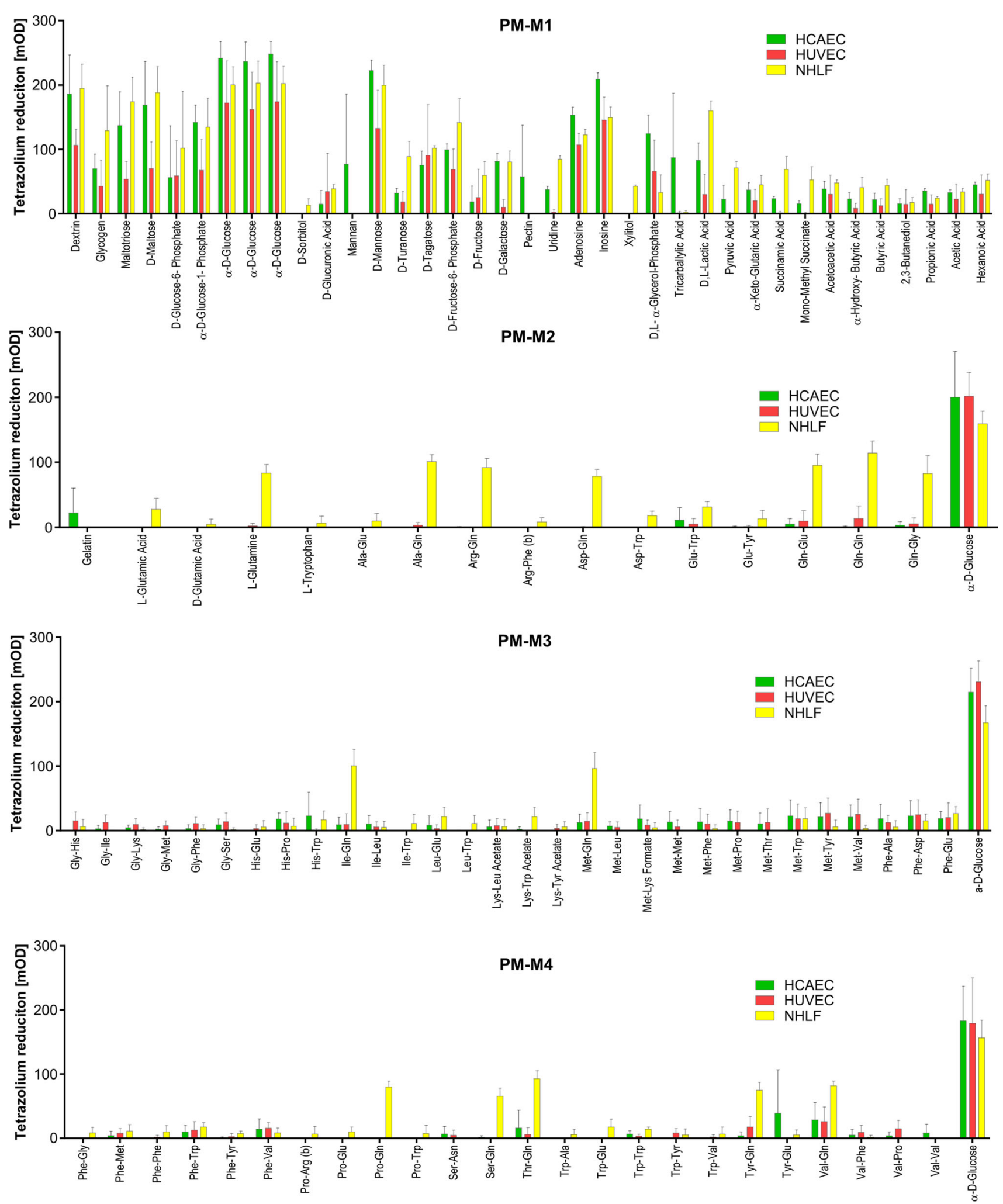

Fig. 4 Comparison of substrate utilization in all three cell types are shown on plates PM-M1 and PM-M2, PM-M3 and PM-M4. Cells were assayed according to the standard protocol and data collected after $24 \mathrm{~h}$ using the OmniLog and PM software, with subtraction of the background. Average height (mOD) of tetrazolium reduction was measured in triplicate. HCAEC human coronary artery endothelial cells, HUVEC human umbilical vein endothelial cells and NHLF normal human lung fibroblasts 
Fig. 5 Unique substrates metabolized exclusively by HCAEC, HUVEC and NHLF, as well as overlapping substrates, are represented by the Venn diagram. HCAEC human coronary artery endothelial cells, HUVEC human umbilical vein endothelial cells and $N H L F$ normal human lung fibroblasts

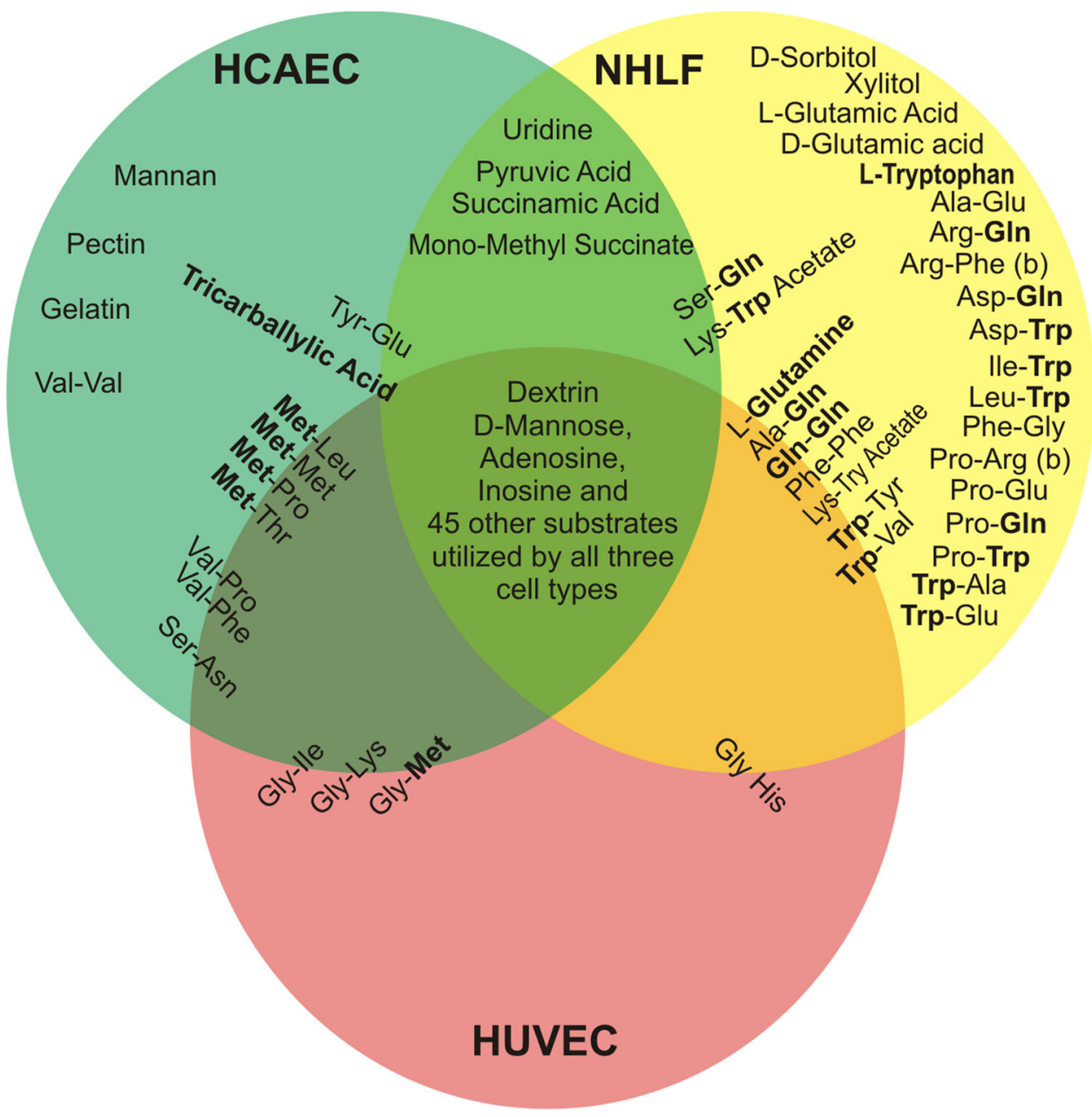

phosphate, D-galactose, pectin, uridine, adenosine, inosine, D, L- $\alpha$-glycerol-phosphate, tricarballylic acid, D, L-lactic acid, pyruvic acid, $\alpha$-keto-glutaric acid, succinamic acid, among others) (Fig. 4). The specific substrates metabolized only by HCAEC, and not by either HUVEC or NHLF were the polymeric substrates mannan, pectin, gelatin, Val-Val and prevalently tricarballylic acid, as shown in Figs. 3 and 4. HUVEC did not show any exclusive metabolism of any substrates, but it was differentiated from HCAEC by notably stronger metabolism of D-glucuronic acid.

The assays indicate that cardiovascular cells, such as HCAEC exhibit extensive metabolic flexibility enabling them to produce energy more rapidly and use the most abundant fuel available, as well. These characteristics might also contribute directly to atherosclerosis and cardiac dysfunction, which are both known to be associated with changes in metabolism and obesity.

NHLF produced saturation of tetrazolium reduction in glucose wells during the $24 \mathrm{~h}$ kinetic measurements and utilized many substrates. Specifically, dextrin, glycogen, maltotriose, D-maltose, D-mannose, D-fructose-6- phosphate, inosine, D, L-lactic acid, D-glucose-6-phosphate, $\alpha$-D-glucose-6-phosphate and adenosine produced strong reductive responses. Strikingly, NHLF also showed higher metabolism of the polyols D-sorbitol and xylitol along with L-glutamic acid, L-glutamine and all of the glutaminecontaining dipeptides (Fig. 4, PM-M2, PM-M3 and PMM4, yellow bars).

The most relevant glutamine-producing tissue is the muscle, accounting for about $90 \%$ of all glutamine synthesized (Newsholme et al. 2003). Glutamine is also released, in small amounts, by the lung and the brain. In contrast, the biggest consumers of glutamine are the cells of intestines (Brosnan 2003), the kidney cells for the acidbase balance, activated immune cells (Newsholme 2001), and many cancer cells (Yuneva et al. 2007). We show for the first time, that normal healthy primary lung fibroblasts (in contrast to either HCAEC or HUVEC) have higher utilization of glutamine and glutamine-associated dipeptides. Glutamine has been shown in the past to be essential for growth of human embryonic diploid lung fibroblasts, when $10 \%$ undialysed calf serum was used as a medium 
supplement (Litwin 1979). Interstitial fibroblasts within a biomatrix are exposed to varying levels of amino acids (Rishikof et al. 1998) and it was previously found that expression of $\alpha 1$ (I) collagen mRNA was directly dependent on amino acid availability (Krupsky et al. 1997). Since type I collagen is a major structural protein in the lung known to participate in tissue fibrosis during systemic sclerosis, the effects of glutamine on its levels would be crucial to study. Rishikof et al. reported in 1998, on the regulation of type I collagen mRNA in human embryonic lung fibroblasts and found that the addition of the combination of glutamine and cysteine increased $\alpha 1(\mathrm{I})$ collagen mRNA levels 6.3-fold (Rishikof et al. 1998). Importantly, glutamine, utilized for nucleotide synthesis (Engstrom and Zetterberg 1984) also increased $\alpha 1(\mathrm{I})$ collagen mRNA in dermal fibroblasts by elevating gene transcription (Bellon et al. 1995).

The tetrazolium reduction measured in L-tryptophan during the $24 \mathrm{~h}$ measurements was detected in low levels only in NHLF. Neither HCAEC nor HUVEC metabolized L-tryptophan during this time frame. It is of interest that the metabolism of L-tryptophan is involved, via the kynurenine pathway, in patients with the eosinophilia-myalgia syndrome (Silver et al. 1992). Ingesting tryptophan may lead to a syndrome characterized by scleroderma-like skin abnormalities, fasciitis, in addition to eosinophilia. Plasma concentrations of L-kynurenine and quinolinic acid, both metabolites of tryptophan, were significantly higher in patients with active disease, as compared to patients studied after eosinophilia had resolved, or normal subjects (Silver et al. 1990). Since in late, diffused scleroderma, lungs can be prevalently affected, it could be hypothesized that this could be due to changed metabolism of tryptophan pathways in lung fibroblasts from patients. This would be relevant to test in the future.

\subsection{Comparison of exclusive and overlapping substrates}

Comparison of all three cell types together revealed that 49 of the 367 tested substrates were utilized by all three cell types, however both HCAEC and NHLF utilized several substrates uniquely, with a distinct pattern. On the other hand, HUVEC did not exhibit any exclusively utilized substrates. The specific substrates metabolized only by HCAEC, and not by HUVEC or NHLF were mannan, pectin, gelatin, Val-Val and prevalently tricarballylic acid, as shown in the Venn diagram (Fig. 5). NHLF were the only cells utilizing D-sorbitol, xylitol, L-glutamic acid, Dglutamic acid, L-tryptophan and the dipeptides Ala-Glu, Arg-Gln, Arg-Phe (b), Asp-Gln, Asp-Trp,Ile-Trp, Leu-Trp, Phe-Gly, Pro-Arg (b), Pro-Glu, Pro-Gln, Pro-Trp, Trp-Ala and Trp-Glu for their energy production (Fig. 5).
Metabolism of substrates was measured within a $24 \mathrm{~h}$ timeframe. In the future, it could be advantageous to measure metabolic activity of different human primary cell lots, passages and preparations at different time periods with altered $\mathrm{CO}_{2}$ and oxygen levels in the incubator. This could be especially important for utilization of certain substrates, such as L-Glutamine, L-Tryptophan, Met- and Val- associated dipeptides, which indicated firm delineation between the fibroblast and endothelial cells.

Our results confirm that human primary cells can exhibit a unique and distinct metabolic fingerprint which could make them differentially susceptible to environmental changes that could subsequently lead to differential pathological processes.

\section{Concluding remarks}

Taken together, this is the first report to date exhibiting specific utilization of different carbon and energy sources of human primary endothelial and fibroblast cells on Biolog PM-M1 to PM-M4 plates. We showed that HCAEC have a higher overall metabolic rate than HUVEC, and that mannan, pectin, gelatin, Val-Val and prevalently tricarballylic acid were utilized exclusively by HCAEC. NHLF exhibited high metabolic activity by utilizing many substrate nutrients for energy production, especially glutamine associated dipeptides (Ala-Gln, ArgGln, Asp-Gln, Gln-Gly, Gln-Gln, Gln-Glu, Ile-Gln, MetGln, Pro-Gln, Ser-Gln, Thr-GlnTyr-Gln and Val-Gln). Also, the lack of utilization of mannan, pectin and gelatin in NHLF, coupled with their unique utilization of D-sorbitol, xylitol, L-tryptophan and tryptophan-associated dipeptides (Asp-Trp, Ile-Trp, Leu-Trp, Pro-Trp, Trp-Ala and Trp-Glu) points to a distinct lung fibroblast metabolic phenotype. Taken together, substrate utilization patterns on PM-M1 to PM-M4 plates provide a baseline metabolic characterization of different human primary cell types, which could be important in distinguishing early and celltype specific metabolic changes that could initiate pathophysiological processes. In the future, it would be crucial to perform further experiments on activated cells or cells isolated from patient tissues, in order to elucidate early cellular changes relevant to cardiac complications, as well as the process of lung fibrosis in systemic sclerosis.

Acknowledgments The authors would like to acknowledge Biolog Inc., Hayward, CA, USA and BIA, d.o.o., Ljubljana, Slovenia for kindly providing the Biolog OmniLog incubator/reader needed to perform the experiments.

Funding This work was supported by the Slovenian Research Agency, with the National Research Program Grant \#P3-0314. 


\section{Compliance with ethical standards}

Conflict of interest The authors state no conflict of interest.

Ethical approval The work was performed within the National Research Program P3-0314, with approval from the Slovene Ethical committee \#99/04/15.

Human and Animal Rights This article does not contain any studies with human participants or animals performed by any of the authors.

\section{References}

Bellon, G., Chaqour, B., Wegrowski, Y., Monboisse, J. C., \& Borel, J. P. (1995). Glutamine increases collagen gene transcription in cultured human fibroblasts. Biochimica et Biophysica Acta, 1268(3), 311-323.

Bochner, B. R., Siri, M., Huang, R. H., Noble, S., Lei, X. H., Clemons, P. A., et al. (2011). Assay of the multiple energyproducing pathways of mammalian cells. PLoS One, 6(3), e18147. doi:10.1371/journal.pone.0018147.

Boueiz, A., Mathai, S. C., Hummers, L. K., \& Hassoun, P. M. (2010). Cardiac complications of systemic sclerosis: recent progress in diagnosis. Current Opinion in Rheumatology, 22(6), 696-703. doi:10.1097/BOR.0b013e32833dfbd8.

Brosnan, J. T. (2003). Interorgan amino acid transport and its regulation. Journal of Nutrition, 133(6 Suppl 1), 2068s-2072s.

Burns, R. L., Rosenberger, P. G., \& Klebe, R. J. (1976). Carbohydrate preferences of mammalian cells. Journal of Cellular Physiology, 88(3), 307-316. doi:10.1002/jcp.1040880306.

Castello-Cros, R., Whitaker-Menezes, D., Molchansky, A., Purkins, G., Soslowsky, L. J., Beason, D. P., et al. (2011). Sclerodermalike properties of skin from caveolin-1-deficient mice: implications for new treatment strategies in patients with fibrosis and systemic sclerosis. Cell Cycle, 10(13), 2140-2150.

Eelen, G., de Zeeuw, P., Simons, M., \& Carmeliet, P. (2015). Endothelial cell metabolism in normal and diseased vasculature. Circulation Research, 116(7), 1231-1244. doi:10.1161/circre saha.116.302855.

Engstrom, W., \& Zetterberg, A. (1984). The relationship between purines, pyrimidines, nucleosides, and glutamine for fibroblast cell proliferation. Journal of Cellular Physiology, 120(2), 233-241. doi:10.1002/jcp.1041200218.

Galvan-Pena, S., \& O’Neill, L. A. (2014). Metabolic reprograming in macrophage polarization. Frontiers in Immunology, 5, 420. doi:10.3389/fimmu.2014.00420.

Goodwin, G. W., \& Taegtmeyer, H. (2000). Improved energy homeostasis of the heart in the metabolic state of exercise. American Journal of Physiology Heart and Circulatory Physiology, 279(4), H1490-H1501.

Hettema, M. E., Zhang, D., de Leeuw, K., Stienstra, Y., Smit, A. J., Kallenberg, C. G., et al. (2008). Early atherosclerosis in systemic sclerosis and its relation to disease or traditional risk factors. Arthritis Research and Therapy, 10(2), R49. doi:10.1186/ar2408.

Kaijser, L., \& Berglund, B. (1992). Myocardial lactate extraction and release at rest and during heavy exercise in healthy men. Acta Physiologica Scandinavica, 144(1), 39-45. doi:10.1111/j.17481716.1992.tb09265.x.

Kolwicz, S. C, Jr, Purohit, S., \& Tian, R. (2013). Cardiac metabolism and its interactions with contraction, growth, and survival of cardiomyocytes. Circulation Research, 113(5), 603-616. doi:10. 1161/circresaha.113.302095.
Krupsky, M., Kuang, P. P., \& Goldstein, R. H. (1997). Regulation of type I collagen mRNA by amino acid deprivation in human lung fibroblasts. Journal of Biological Chemistry, 272(21), 13864-13868.

Kuwana, M., Okazaki, Y., Yasuoka, H., Kawakami, Y., \& Ikeda, Y. (2004). Defective vasculogenesis in systemic sclerosis. Lancet, 364(9434), 603-610. doi:10.1016/S0140-6736(04)16853-0.

Lakota, K., Mrak-Poljsak, K., Bozic, B., Tomsic, M., \& Sodin-Semrl, S. (2013). Serum amyloid A activation of human coronary artery endothelial cells exhibits a neutrophil promoting molecular profile. Microvascular Research, 90, 55-63. doi:10.1016/j.mvr. 2013.07.011.

Lakota, K., Mrak-Poljsak, K., Rozman, B., Kveder, T., Tomsic, M., \& Sodin-Semrl, S. (2007). Serum amyloid A activation of inflammatory and adhesion molecules inhuman coronary artery and umbilicalvein endothelial cells. European Journal of Inflammation, 5(2), 73-81.

Lakota, K., Mrak-Poljsak, K., Rozman, B., \& Sodin-Semrl, S. (2009). Increased responsiveness of human coronary artery endothelial cells in inflammation and coagulation. Mediators of Inflammation, 2009, 146872. doi:10.1155/2009/146872.

Litwin, J. (1979). Necessary amino acids and vitamins for the growth of human diploid fibroblasts. Journal of Cell Science, 40, 281-291.

Meune, C., Vignaux, O., Kahan, A., \& Allanore, Y. (2010). Heart involvement in systemic sclerosis: evolving concept and diagnostic methodologies. Archives of Cardiovascular Diseases, 103(1), 46-52. doi:10.1016/j.acvd.2009.06.009.

Newsholme, P. (2001). Why is L-glutamine metabolism important to cells of the immune system in health, postinjury, surgery or infection? The Journal of Nutrition, 131(9 Suppl), 2515S-2522S. discussion 2523S-2514S.

Newsholme, P., Lima, M. M., Procopio, J., Pithon-Curi, T. C., Doi, S. Q., Bazotte, R. B., et al. (2003). Glutamine and glutamate as vital metabolites. Brazilian Journal of Medical and Biological Research, 36(2), 153-163.

Ngian, G. S., Sahhar, J., Wicks, I. P., \& Van Doornum, S. (2011). Cardiovascular disease in systemic sclerosis-an emerging association? Arthritis Research and Therapy, 13(4), 237. doi:10. 1186/ar3445.

Pavlides, S., Tsirigos, A., Vera, I., Flomenberg, N., Frank, P. G., Casimiro, M. C., et al. (2010). Transcriptional evidence for the "Reverse Warburg Effect" in human breast cancer tumor stroma and metastasis: similarities with oxidative stress, inflammation, Alzheimer's disease, and "neuron-glia metabolic coupling". Aging (Albany NY), 2(4), 185-199.

Pavlides, S., Whitaker-Menezes, D., Castello-Cros, R., Flomenberg, N., Witkiewicz, A. K., Frank, P. G., et al. (2009). The reverse Warburg effect: aerobic glycolysis in cancer associated fibroblasts and the tumor stroma. Cell Cycle, 8(23), 3984-4001.

Pearson, H. (2007). Meet the human metabolome. Nature, 446(7131), 8. doi:10.1038/446008a.

Putluri, N., Shojaie, A., Vasu, V. T., Nalluri, S., Vareed, S. K., Putluri, V., et al. (2011). Metabolomic profiling reveals a role for androgen in activating amino acid metabolism and methylation in prostate cancer cells. PLoS One, 6(7), e21417. doi:10.1371/ journal.pone.0021417.

Reiseter, S., Molberg, O., Gunnarsson, R., Lund, M. B., Aalokken, T. M., Aukrust, P., et al. (2015). Associations between circulating endostatin levels and vascular organ damage in systemic sclerosis and mixed connective tissue disease: an observational study. Arthritis Research and Therapy, 17, 231. doi:10.1186/ s13075-015-0756-5.

Rishikof, D. C., Kuang, P. P., Poliks, C., \& Goldstein, R. H. (1998). Regulation of type I collagen mRNA in lung fibroblasts by cystine availability. Biochemical Journal, 331(Pt 2), 417-422. 
Rosenberg, R. D., \& Aird, W. C. (1999). Vascular-bed-specific hemostasis and hypercoagulable states. New England Journal of Medicine, 340(20), 1555-1564. doi:10.1056/NEJM19990520 3402007.

Schonekess, B. O. (1997). Competition between lactate and fatty acids as sources of ATP in the isolated working rat heart. Journal of Molecular and Cellular Cardiology, 29(10), 2725-2733. doi:10.1006/jmcc.1997.0504.

Silver, R. M., Heyes, M. P., Maize, J. C., Quearry, B., VionnetFuasset, M., \& Sternberg, E. M. (1990). Scleroderma, fasciitis, and eosinophilia associated with the ingestion of tryptophan. New England Journal of Medicine, 322(13), 874-881. doi:10. 1056/nejm199003293221302.

Silver, R. M., McKinley, K., Smith, E. A., Quearry, B., Harati, Y., Sternberg, E. M., et al. (1992). Tryptophan metabolism via the kynurenine pathway in patients with the eosinophilia-myalgia syndrome. Arthritis and Rheumatism, 35(9), 1097-1105.

Usategui, A., del Rey, M. J., \& Pablos, J. L. (2011). Fibroblast abnormalities in the pathogenesis of systemic sclerosis. Expert
Review Clinical Immunology, 7(4), 491-498. doi:10.1586/eci.11. 39.

Vallerie, S. N., \& Bornfeldt, K. E. (2015). Metabolic flexibility and dysfunction in cardiovascular cells. Arteriosclerosis, Thrombosis, and Vascular Biology, 35(9), e37-e42. doi:10.1161/ATV BAHA.115.306226.

van Laar, J. M., \& Varga, J. (2015). The immunopathology of systemic sclerosis. Seminars in Immunopathology, 37(5), 439-441. doi:10.1007/s00281-015-0517-1.

Wentz, A. E., d'Avignon, D. A., Weber, M. L., Cotter, D. G., Doherty, J. M., Kerns, R., et al. (2010). Adaptation of myocardial substrate metabolism to a ketogenic nutrient environment. Journal of Biological Chemistry, 285(32), 24447-24456. doi:10.1074/jbc.M110.100651.

Yuneva, M., Zamboni, N., Oefner, P., Sachidanandam, R., \& Lazebnik, Y. (2007). Deficiency in glutamine but not glucose induces MYC-dependent apoptosis in human cells. Journal of Cell Biology, 178(1), 93-105. doi:10.1083/jcb.200703099. 